\title{
The study of $\gamma / \phi_{3}$-sensitive hadronic decays at LHCb
}

\section{Angelo Carbone*t}

Istituto Nazionale di Fisica Nucleare, Sezione di Bologna

E-mail: angelo.carbone@bo.infn.it

We report preliminary measurements on charmless charged two-body and open-charm charged B hadron decay modes, using an integrated luminosity of $0.32 \mathrm{fb}^{-1}$ collected by LHCb in the first part of the 2011 run, the direct $C P$ asymmetries $A_{C P}\left(B^{0} \rightarrow K \pi\right)=-0.088 \pm 0.011 \pm 0.008$ and $A_{C P}\left(B_{s}^{0} \rightarrow \pi K\right)=0.27 \pm 0.08 \pm 0.02$ is measured. Furthermore, the branching fractions $B R\left(B^{0} \rightarrow K^{+} K^{-}\right)=\left(0.13_{-0.05}^{+0.06} \pm 0.07\right) \times 10^{-6}$ and $B R\left(B_{s}^{0} \rightarrow \pi^{+} \pi^{-}\right)=\left(0.98_{-0.19}^{+0.23} \pm 0.11\right) \times 10^{-6}$ is measured. Using an integrated luminosity of $37 \mathrm{pb}^{-1}$ collected by LHCb in 2010 , the ratio of two $B_{u}$ hadronic branching fractions $B R\left(B^{ \pm} \rightarrow D K^{ \pm}\right) / B R\left(B^{ \pm} \rightarrow D \pi^{ \pm}\right)$is measured for events in which the $D$ meson is reconstructed in several final states. Using the Cabibbo-favored decays $D^{0} \rightarrow K^{-} \pi^{+}$and $D^{0} \rightarrow K^{-} \pi^{+} \pi^{-} \pi^{+}$, the ratio is measured as $(6.30 \pm 0.38 \pm 0.40) \%$. It is determined to be $(9.31 \pm 1.89 \pm 0.53) \%$ in the $C P$ eigenstate mode $D \rightarrow K^{+} K^{-}$and $\left(12_{-5}^{+6} \pm 1\right) \%$ in the $D \rightarrow K_{s}^{0} \pi^{+} \pi^{-}$channel. We also provide a preliminary value of the $\gamma$-sensitive observable: $R_{C P+}=1.48 \pm 0.31 \pm 0.12$.

The 2011 Europhysics Conference on High Energy Physics-HEP 2011,

July 21-27, 2011

Grenoble, Rhône-Alpes France

\footnotetext{
*Speaker.

${ }^{\dagger}$ On behalf of LHCb Collaboration
} 


\section{Introduction}

In this paper we present preliminary results based on the reconstruction of charmless charged two-body and open-charm charged B hadron decay modes.

The charmless charged two-body decays, $H_{b} \rightarrow h^{+} h^{\prime-}$, comprise a large set of modes, namely: $B^{0} \rightarrow \pi^{+} \pi^{-}, B^{0} \rightarrow K^{+} \pi^{-}, B_{s}^{0} \rightarrow K^{+} K^{-}, B_{s}^{0} \rightarrow \pi^{+} K^{-}, \Lambda_{b} \rightarrow p K^{-}, \Lambda_{b} \rightarrow p \pi^{-}, B^{0} \rightarrow K^{+} K^{-}$, $B_{s}^{0} \rightarrow \pi^{+} \pi^{-}$plus their $C P$-conjugate states. Such decays provide sensitive probes of the CabibboKobayashi-Maskawa [1] matrix and have the potential to reveal the presence of New Physics [2]. In the following, we will present the preliminary measurements of the direct $C P$ asymmetries in the $B^{0} \rightarrow K \pi$ and $B_{s}^{0} \rightarrow \pi K$ decays, defined in terms of decay rates as

$$
A_{C P}\left(B^{0} \rightarrow K \pi\right)=\frac{\Gamma\left(\bar{B}^{0} \rightarrow K^{-} \pi^{+}\right)-\Gamma\left(B^{0} \rightarrow K^{+} \pi^{-}\right)}{\Gamma\left(\bar{B}^{0} \rightarrow K^{-} \pi^{+}\right)+\Gamma\left(B^{0} \rightarrow K^{+} \pi^{-}\right)}
$$

and

$$
A_{C P}\left(B_{s}^{0} \rightarrow \pi K\right)=\frac{\Gamma\left(\bar{B}_{s}^{0} \rightarrow \pi^{-} K^{+}\right)-\Gamma\left(B_{s}^{0} \rightarrow \pi^{+} K^{-}\right)}{\Gamma\left(\bar{B}_{s}^{0} \rightarrow \pi^{-} K^{+}\right)+\Gamma\left(B_{s}^{0} \rightarrow \pi^{+} K^{-}\right)} .
$$

We also provide measurements of the branching fractions $B R\left(B^{0} \rightarrow K^{+} K^{-}\right)$and $B R\left(B_{s}^{0} \rightarrow \pi^{+} \pi^{-}\right)$. These results have been obtained using the data collected by LHCb during the first part of 2011 at a centre of mass energy of $7 \mathrm{TeV}$, corresponding to an integrated luminosity of $320 \mathrm{pb}^{-1}$.

In addition, we report preliminary results based on the reconstruction of open-charm charged B decays, $B^{ \pm} \rightarrow D K^{ \pm}$, where the $D$ is reconstructed in several final states. They can be used to provide an unambiguously extraction of $\gamma$ by measuring the partial width and the charge asymmetries [3]. Using $37 \mathrm{pb}^{-1}$ of data collected by LHCb in the 2010, we provide the measurement of branching fraction of the $B^{+} \rightarrow D K^{+}$decay with respect to the Cabibbo-favored (CF) $B^{+} \rightarrow D \pi^{+}$, simultaneously in the $D \rightarrow K^{-} \pi^{+}, D \rightarrow K^{-} \pi^{+} \pi^{-} \pi^{+}$modes. This ratio, $R_{C F}^{K / \pi}$, has been measured several times by previous experiments but some tensions exist in their results [4]. The ratio of the branching fractions is also measured in the $C P+$ eigenstate mode $D \rightarrow K^{+} K^{-}\left(R_{C P+}^{K / \pi}\right)$ and the multibody $D \rightarrow K_{s}^{0} \pi^{+} \pi^{-}$final state $\left(R_{K_{s}^{0} \pi \pi}^{K / \pi}\right)$. We also provide a preliminary value of the $\gamma$-sensitive observable $R_{C P+}$. For more details see also [5].

\section{The LHCb detector}

The $\mathrm{LHCb}$ detector [6] is a single arm spectrometer in the forward direction. It is composed of a vertex detector around the interaction region, a set of tracking stations in front of and behind a dipole magnet that provides a field integral of $4 \mathrm{Tm}$, two Ring-Imaging Cherenkov (RICH) detectors, electromagnetic and hadronic calorimeters complemented with pre-shower and scintillating pad detectors, and a set of muon chambers. The two RICH detectors are of particular importance for this analysis, as they provide the particle identification (PID) information needed to disentangle the various $H_{b} \rightarrow h^{+} h^{\prime-}$ and $B^{ \pm} \rightarrow D h^{ \pm}$final states. They are able to separate efficiently $\pi, K$ and protons in a momentum range from $2 \mathrm{GeV} / c$ up to and beyond $100 \mathrm{GeV} / c$. RICH-1 is installed in front of the magnet and uses areogel and $\mathrm{C}_{4} \mathrm{~F}_{10}$ as radiators, while RICH-2 is installed behind the magnet and employs $\mathrm{CF}_{4}$. 


\section{Event selection}

The decays $H_{b} \rightarrow h^{+} h^{\prime-}$ and $B^{ \pm} \rightarrow D K^{ \pm}$are principally selected by the two-level hadronic trigger of LHCb. The first level (Level 0), an hardware trigger, selects events with high transverse energy clusters in the hadronic calorimeter. The second level, the so called High Level Trigger (HLT), is software-based and selects events with at least one track with high transverse momentum and large impact parameter with respect to all reconstructed primary vertices.

The events used in these analyses are extracted from the triggered data using different offline selections, each one targeted to achieve the best sensitivity on the measurements of interest.

\section{Calibration of particle identification}

The calibration of the PID observables is a crucial aspect of these analyses, as it is the only variable allowing us to discriminate between the various decay modes. Hence, in order to determine the amount of cross-feed backgrounds for a given channel, the relative efficiencies of the PID selection cuts, employed to identify the specific final state of interest, play a key role.

Thanks to the high production rate of $D^{*}$ mesons at LHC and to the kinematic characteristics of $D^{*+} \rightarrow D^{0}\left(K^{-} \pi^{+}\right) \pi^{+}$decay chain (and its charge conjugate), samples of large statistics and high purity of $\pi$ and $K$ can be extracted from these events without any use of PID information. Then, the efficiency of the PID cut has been estimated by re-weighting the distributions of PID observables in relevant kinematic variables, in order to match the corresponding distributions of particles from $B$-hadron. In the case of the $H_{b} \rightarrow h^{+} h^{\prime-}$ decays, cross-feed backgrounds are also characterized by the presence of protons in the final state (i.e. $\Lambda_{b} \rightarrow p \pi, p K$ ), in this case the calibration has been realized by means of a sample of $\Lambda \rightarrow p \pi^{-}$decays.

\section{Signal extraction}

The $B^{+} \rightarrow D K^{+}$and $B^{+} \rightarrow D \pi^{+}$signal events are extracted with an unbinned maximum likelihood fit to the mass distribution of the $\mathrm{B}$ candidates. $B^{+} \rightarrow D K^{+}$events are separated from $B^{+} \rightarrow D \pi^{+}$with a PID requirement on the bachelor. As example the result of the fit is shown in Fig. 1 for $D \rightarrow K \pi$.

The $B^{0} \rightarrow K \pi, B_{s}^{0} \rightarrow \pi K, B^{0} \rightarrow K K$ and $B_{s}^{0} \rightarrow \pi \pi$ signal events are extracted with an unbinned maximum likelihood fit to different final state mass spectra of events passing the offline selections. The various final states are separated using PID requirements in order to have exclusive categories corresponding to distinct final state hypotheses, namely $K^{+} \pi^{-}, K^{-} \pi^{+}, K^{+} K^{-}$and $\pi^{+} \pi^{-}$. In the fits the amount of background for a given channel, due to the other channels where at least one particle has been mis-identified (cross-feed background), has been taken into account. The result of the fit is shown on Figs. 2, 3. 

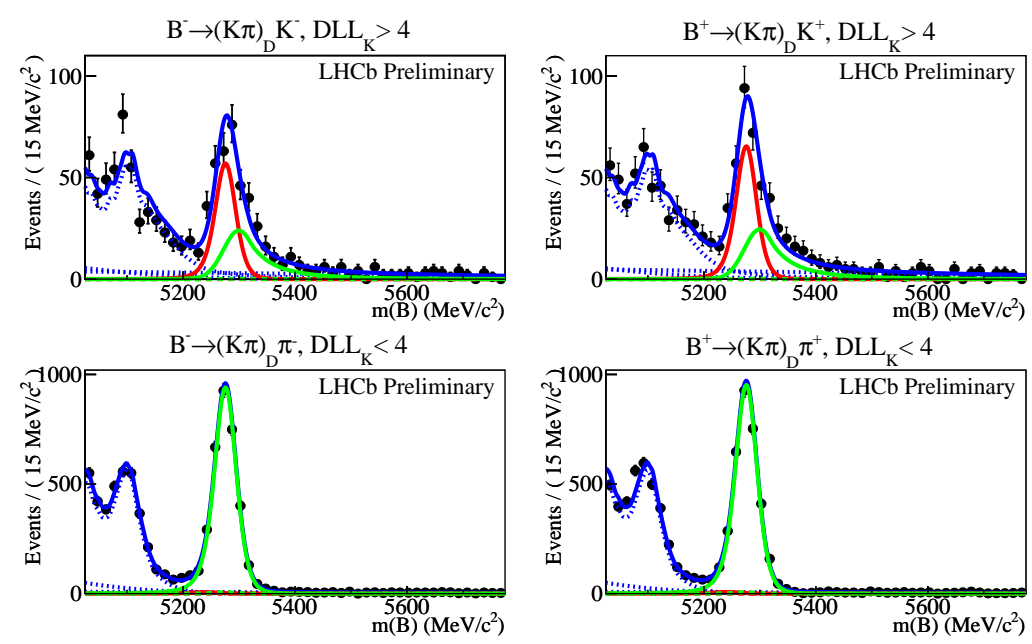

Figure 1: $B^{ \pm} \rightarrow D h^{ \pm}$distributions for $D \rightarrow K \pi$. The top plots contain the candidates that pass the bachelor track's PID requirement and are reconstructed with a kaon mass in the invariant-mass sum. The bottom row candidates fail this requirement and are assigned a pion mass. The solid red curve is $B^{ \pm} \rightarrow D K^{ \pm}$and the solid green curve is $B^{ \pm} \rightarrow D \pi^{ \pm}$. The charmless components (if present) are shown as dashed red and green lines. The combinatoric, partially-reconstructed and semi-leptonic backgrounds are shown by blue dashed lines. The sum of the component PDFs is represented by the solid blue line.
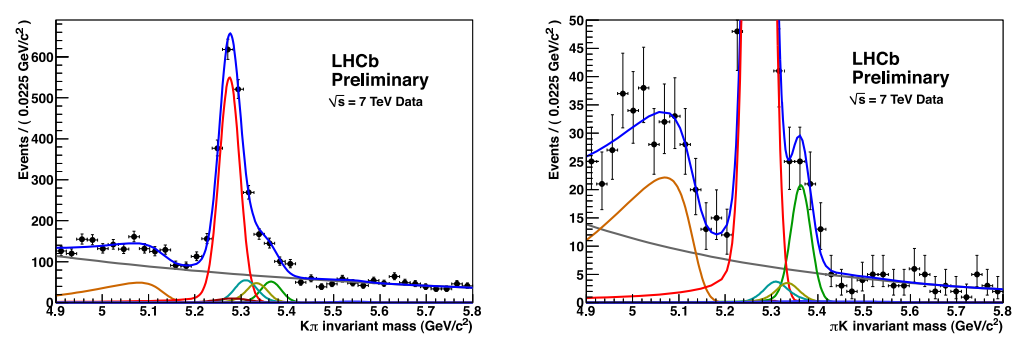

Figure 2: $K^{+} \pi^{-}$(plus charge conjugate) invariant mass spectrum for events surviving the event selection optimized for the best sensitivity on $A_{C P}\left(B^{0} \rightarrow K^{+} \pi^{-}\right)$(left) and $A_{C P}\left(B^{0} \rightarrow K^{+} \pi^{-}\right)$(right). The result of the unbinned maximum likelihood fit is superimposed. The main components contributing to the fit model explained in the text are also visible: $B^{0} \rightarrow K \pi$ (red), wrong sign $B^{0} \rightarrow K \pi$ combination (dark red), $B^{0} \rightarrow \pi^{+} \pi^{-}$(light blue), $B_{s}^{0} \rightarrow K^{+} K^{-}$(dark yellow), $B_{s}^{0} \rightarrow \pi K$ (green), combinatorial background (grey), 3-body partially reconstructed decays (orange).

\section{Results and Conclusions}

Using data collected by the $\mathrm{LHCb}$ detector during the fist part of 2011 run we provide preliminary values of the direct $\mathrm{CP}$ asymmetries: $A_{C P}\left(B^{0} \rightarrow K \pi\right)=-0.088 \pm 0.011 \pm 0.008$ and $A_{C P}\left(B_{s}^{0} \rightarrow \pi K\right)=0.27 \pm 0.08 \pm 0.02$. Our result for $A_{C P}\left(B^{0} \rightarrow K \pi\right)$ constitutes the most precise measurement in the world, and is in good agreement with the current world average provided by HFAG [7]: $A_{C P}\left(B^{0} \rightarrow K^{+} \pi^{-}\right)=-0.098_{-0.011}^{+0.012}$. Our result for $A_{C P}\left(B_{s}^{0} \rightarrow \pi K\right)$ is the first evidence of $C P$ violation in the $B_{s}^{0} \rightarrow \pi K$ decay, and is in agreement with the only measurement currently available, performed by $\mathrm{CDF}$ [9]: $A_{C P}\left(B_{s}^{0} \rightarrow \pi^{+} K^{-}\right)=0.39 \pm 0.15 \pm 0.08$.

We also provide preliminary values of the relative branching ratios: $B R\left(B^{0} \rightarrow K^{+} K^{-}\right)=$ 

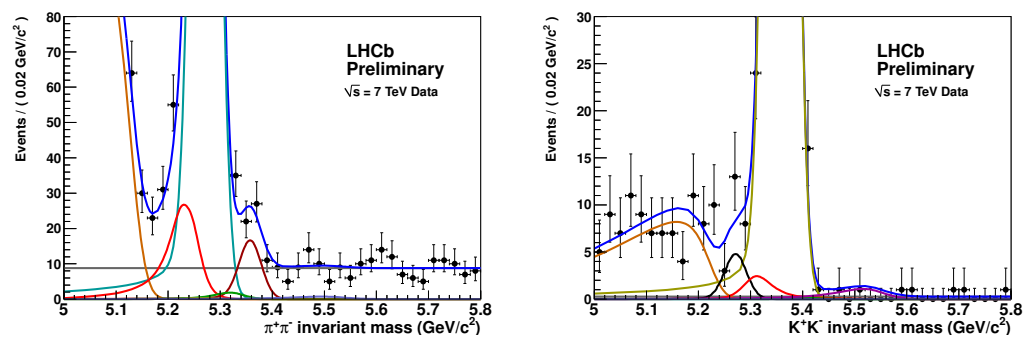

Figure 3: $\pi^{+} \pi^{-}$(left) and $K^{+} K^{-}$invariant mass spectrum for events surviving the offline event selection. The result of the unbinned maximum likelihood fit is superimposed. The main components contributing to the fit model are also visible: $B_{s}^{0} \rightarrow \pi^{+} \pi^{-}$(brown), $B^{0} \rightarrow \pi^{+} \pi^{-}$(light blue), and $B_{s}^{0} \rightarrow \pi K$ (green), visible only in the left plot; $B^{0} \rightarrow K^{+} K^{-}$(black), $B_{s}^{0} \rightarrow K^{+} K^{-}$(dark yellow), and $\Lambda_{b} \rightarrow p K$ (purple), visible only in the right plot; $B^{0} \rightarrow K \pi$ (red), combinatorial background (grey) and 3-body partially reconstructed decays (orange) present in both plots.

$\left(0.13_{-0.05}^{+0.06} \pm 0.07\right) \times 10^{-6}$ and $B R\left(B_{s}^{0} \rightarrow \pi^{+} \pi^{-}\right)=\left(0.98_{-0.19}^{+0.23} \pm 0.11\right) \times 10^{-6}$. In particular, we find a total significance of $5.3 \sigma$ for the $B_{s}^{0} \rightarrow \pi^{+} \pi^{-}$signal, which is observed for the first time. Our values are in agreement with the recent CDF results [8]: $B R\left(B^{0} \rightarrow K^{+} K^{-}\right)=(0.23 \pm 0.10 \pm 0.10) \times 10^{-6}$ and $B R\left(B_{s}^{0} \rightarrow \pi^{+} \pi^{-}\right)=(0.57 \pm 0.15 \pm 0.10) \times 10^{-6}$.

The ratio of the $B^{ \pm} \rightarrow D K^{ \pm}$branching fraction to that of $B^{ \pm} \rightarrow D \pi^{ \pm}$is measured for several $D$ decays using the $2010 \mathrm{LHCb}$ dataset: $R_{C F}^{K / \pi}=(6.30 \pm 0.38 \pm 0.40) \%, R_{C P+}^{K / \pi}=(9.31 \pm 1.89 \pm$ $0.53) \%$ and $R_{K_{S} \pi \pi}^{K / \pi}=\left(12.0_{-5.0}^{+6.0} \pm 1.0\right) \%$. From the ratio of the first two results above, the following $\gamma$-sensitive observable is deduced $R_{C P+}=1.48 \pm 0.31 \pm 0.12$.

\section{References}

[1] N. Cabibbo, Phys. Rev. Lett. 10 (1963) 531.

M. Kobayashi and T. Maskawa, Prog. Theor. Phys. 49 (1973) 652.

[2] R. Fleischer, Phys. Lett. B 459 (1999) 306

R. Fleischer, Eur. Phys. J. C 52 (2007) 267

R. Fleischer and R. Knegjens, arXiv:1011.1096 [hep-ph].

[3] M. Gronau and D. London, Phys. Lett. B 253 (1991) 483

M. Gronau and D. Wyler, Phys. Lett. B 265 (1991) 172

I. Dunietz, Phys. Lett. B 270 (1991) 75

[4] K. Nakamura et al. [Particle Data Group], J. Phys. G 37 (2010) 075021

[5] LHCb Collaboration, LHCb-CONF-2011-042, LHCb-CONF-2011-031

[6] A. A. Alves et al. [LHCb Collaboration], JINST 3 (2008) S08005.

[7] D. Asner et al. [Heavy Flavor Averaging Group Collaboration], arXiv:1010.1589 [hep-ex].

[8] T. Aaltonen et al. [CDF Collaboration], arXiv:hep-ex/11110485.

[9] T. Aaltonen et al. [CDF Collaboration], Phys. Rev. Lett. 106 (2011) 181802 arXiv:1103.5762 [hep-ex]. 